\title{
Site-specific modifications to AAV8 capsid yields enhanced brain transduction in the neonatal MPS IIIB mouse
}

\author{
Janine A. Gilkes ${ }^{1} \cdot$ Benjamin L. Judkins $\mathbb{D}^{1} \cdot$ Brontie N. Herrera $^{1} \cdot$ Ronald J. Mandel ${ }^{2} \cdot$ Sanford L. Boye ${ }^{3}$. \\ Shannon E. Boye ${ }^{4} \cdot$ Arun Srivastava $^{4} \cdot$ Coy D. Heldermon $\mathbb{B}^{1}$
}

Received: 2 April 2020 / Revised: 3 September 2020 / Accepted: 19 October 2020 / Published online: 26 November 2020

(c) The Author(s), under exclusive licence to Springer Nature Limited 2020

\begin{abstract}
Mucopolysaccharidosis type IIIB (MPS IIIB) is an autosomal recessive lysosomal disease caused by defective production of the enzyme $\alpha-\mathrm{N}$-acetylglucosaminidase. It is characterized by severe and complex central nervous system degeneration. Effective therapies will likely target early onset disease and overcome the blood-brain barrier. Modifications of adenoassociated viral (AAV) vector capsids that enhance transduction efficiency have been described in the retina. Herein, we describe for the first time, a transduction assessment of two intracranially administered adeno-associated virus serotype 8 variants, in which specific surface-exposed tyrosine $(\mathrm{Y})$ and threonine $(\mathrm{T})$ residues were substituted with phenylalanine $(\mathrm{F})$ and valine $(\mathrm{V})$ residues, respectively. A double-mutant $(\mathrm{Y} 444+733 \mathrm{~F})$ and a triple-mutant $(\mathrm{Y} 444+733 \mathrm{~F}+\mathrm{T} 494 \mathrm{~V})$ AAV8 were evaluated for their efficacy for the potential treatment of MPS IIIB in a neonatal setting. We evaluated biodistribution and transduction profiles of both variants compared to the unmodified parental AAV8, and assessed whether the method of vector administration would modulate their utility. Vectors were administered through four intracranial routes: six sites (IC6), thalamic (T), intracerebroventricular, and ventral tegmental area into neonatal mice. Overall, we conclude that the IC6 method resulted in the widest biodistribution within the brain. Noteworthy, we demonstrate that GFP intensity was significantly more robust with AAV8 (double $\mathrm{Y}-\mathrm{F}+\mathrm{T}-\mathrm{V}$ ) compared to AAV8 (double $\mathrm{Y}-\mathrm{F}$ ). This provides proof of concept for the enhanced utility of IC6 administration of the capsid modified AAV8 (double Y-F + T-V) as a valid therapeutic approach for the treatment of MPS IIIB, with further implications for other monogenic diseases.
\end{abstract}

\section{Introduction}

One critical component of effective brain delivery is widespread delivery of therapeutic products throughout the central nervous system (CNS), as most of these disorders diffusely affect the CNS $[1,2]$. Therefore, identifying a gene transfer

$\triangle$ Coy D. Heldermon

coy.heldermon@medicine.ufl.edu

1 Department of Medicine, University of Florida College of Medicine, Gainesville, FL, USA

2 Department of Neuroscience, University of Florida College of Medicine, Gainesville, FL, USA

3 Department of Pediatrics, Powell Gene Therapy Center, University of Florida College of Medicine, Gainesville, FL, USA

4 Department of Pediatrics, Division of Cellular and Molecular Therapy, University of Florida College of Medicine, Gainesville, FL, USA injection method and vector that will diffuse through the brain more effectively is critical for any disorder that results from somatic mutations that are ubiquitous. The gene therapy results obtained in models of an array of autosomal recessive lysosomal disorders that predominantly affect the brain indicate that more widespread transduction and protein expression will result in better therapeutic effects in vivo. In addition, identifying the most efficient transduction regimen should also result in a safer therapeutic strategy. The requirement for efficient viral transduction is especially true of lysosomal disorders whose most devastating symptoms are the result of widespread brain pathology. Given that currently available recombinant adeno-associated viral vectors (rAAV) do not globally transduce the brain after peripheral injection, there are two basic parameters that can be modified to improve distribution of brain transduction: improving the efficiency of vector transduction via capsid alterations and/or choosing the most efficient route of injection. Recent studies, aimed at improving our understanding of the cellular roadblocks affecting the efficiency of adeno-associated viral (AAV) 
transduction, revealed that the ubiquitin-proteasome pathway plays an essential role in AAV2 intracellular trafficking [3-5]. This is mediated at least in part by epidermal growth factor receptor protein tyrosine kinase (EGFR-PTK). Additionally a host cell protein, FK506-binding protein 52 (FKBP52) in its tyrosine-phosphorylated form, prevents viral second-strand DNA synthesis, resulting in inhibition of AAV-mediated transgene expression [6]. Both the viral capsid and human FKBP52 protein can be phosphorylated by EGFR-PTK [7]. This effect results in substantial numbers of ubiquitinated virions being recognized and targeted for proteosomal degradation on their way to the nucleus, and inefficient second-strand synthesis, thus leading to inefficient nuclear transport. Phosphorylation prior to ubiquitination can occur at tyrosine, serine, or threonine residues. Therefore, substitution of surface-exposed tyrosine or threonine residues on AAV2 capsids was undertaken to allow the vectors to escape ubiquitination and subsequent degradation. Importantly, mutagenesis of highly conserved exposed tyrosine residues (Y444F, Y500F, or Y730F) on AAV2 capsids enhanced transduction up to tenfold in HeLa cells and 30-fold in mouse liver [8]. Since then, single or combined tyrosine mutants of AAV2 have been successfully tested in vitro in fibroblasts and mesenchymal stem cells [9] and in vivo in murine hepatocytes [10], and the retina [3]. Improved transduction of mouse skeletal muscle was also obtained with tyrosine mutants of AAV8 in the lungs [11] and in the skeletal muscle by AAV6 vectors [12]. In our previous studies, we compared neonatal intracranial administration of AAV5, -8, -9, and $-\mathrm{rh} 10$ and concluded that AAV8 was superior to AAV5, -9, and rh10 in its ability to foster robust and widespread transduction within the mucopolysaccharidosis type IIIB (MPS IIIB) brain $[13,14]$. Consistent with previous studies, we also showed that AAV8 expressed a preference for neurons and astrocytes when injected in neonates. To improve upon the therapeutic capacity of AAV8, we capitalized on the improved transduction efficiency of capsid modified vectors in somatic organs. The use of AAV8 capsid tyrosine mutants in the brain of MPS IIIB animals has thus far not been assessed. In particular, based on its efficiency in a retinal model, we selected AAV8 (double Y-F) and AAV8 (double Y-F + T-V) for further analysis.

To address the variable of the most efficient injection route to transduce the $\mathrm{CNS}$, the aforementioned modified rAAV8 vectors were administered either via an intraparenchymal six site (IC6) route, an intracerebroventricular (ICV) injection, intra-thalamic (TH), and ventral tegmental area (VTA) methods. As the six site method, which we have tested previously, is more invasive, requiring six burr holes, our goal is to identify an alternate less invasive method, which would lead to the same level of global brain biodistribution. The four ventricular spaces in the brain are filled with $\mathrm{CSF}$, which bathes the brain and spinal cord and protects these structures from injury. As this represents a promising route to achieve global brain coverage, several groups have attempted to exploit the ventricular system as a therapeutic approach. However, this approach displayed mixed results, often as a consequence of animal age and AAV serotype. Similarly, direct intraparenchymal administration of rAAV has also been used to achieve widespread transduction. The inclusion of the thalamic and VTA injection sites is based on the widespread projection pattern of these anatomical areas. The thalamus projects to the entire cerebral cortex, while the VTA projects to the frontal cortex and basal ganglia. Since lysosomal enzymes can be functionally transported between cells via mannose-6phosphate receptors, these small injection sites may affect large areas of the brain.

To address these issues in the context of the MPS IIIB model, we performed a comprehensive analysis of two capsid modified AAV8 variants compared to parental AAV8 when administered via IC6, ICV, TH, or VTA methods to identify the most efficient vector and optimal administration route. We observed a clearly superior transduction area and intensity with the IC6 AAV8 (double $\mathrm{Y}-\mathrm{F}+\mathrm{T}-\mathrm{V})$. We anticipate these results will contribute to clinical approaches by identifying the optimal gene delivery vector and method of delivery for treatment of MPS IIIB.

\section{Results}

\section{Capsid modification results in varying effect on brain transduction}

We first sought to establish whether any differences existed between the different capsid modified AAV8 variants compared to the parental unmodified AAV8. We have previously shown that intracranial six site administration (IC6) of AAV8 leads to near global brain biodistribution within the CNS. We therefore initially assessed for changes in transduction efficiency as a consequence of capsid modification via IC6 administration. We qualitatively investigated four structural areas of functional significance within the brain for transduction. Surprisingly, we saw that the AAV8 (double Y-F) modified vector resulted in inefficient cellular transduction compared to unmodified AAV8. However, use of AAV8 (double $\mathrm{Y}-\mathrm{F}+\mathrm{T}-\mathrm{V}$ ) resulted in robust transduction of the cortex and hippocampus, with good transduction of the thalamus and cerebellum (Fig. 1). To gauge the difference in GFP intensity as a consequence of capsid modification and mitigate the effects of GFP saturation in florescence signaling, we analyzed the cortical, hippocampal, thalamic, and cerebellar regions of midsagittal brain sections of animals treated with each vector using a near-infrared dye. When data were normalized to 


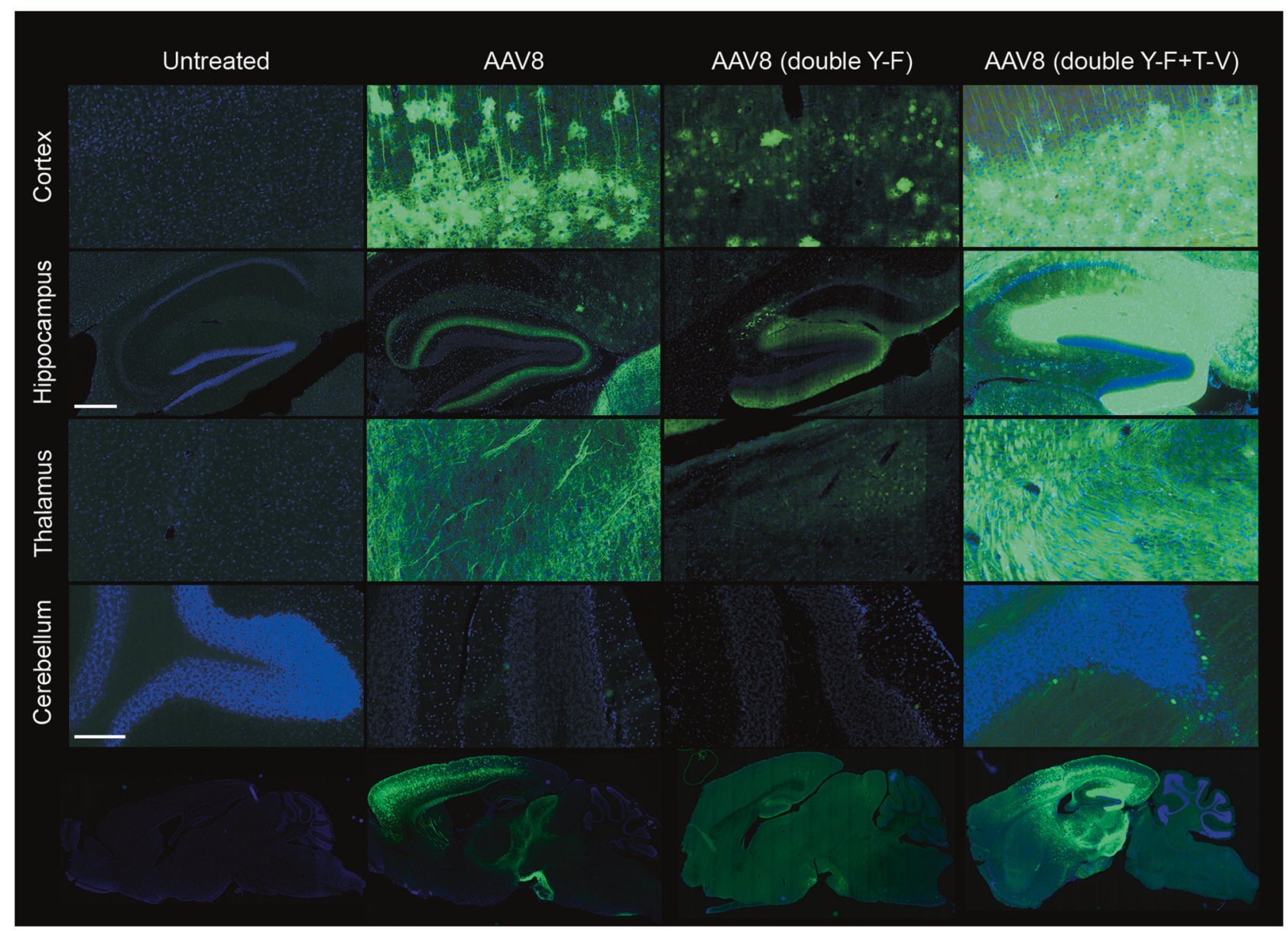

Fig. 1 Assessment of transduction efficiency of AAV8, AAV8 (double Y-F), and AAV8 (double Y-F + T-V) when injected via the IC6 method. Three-month-old MPS IIIB mice were assessed for GFP expression and tissue penetration into the cortex, hippocampus, thalamus, and cerebellum. Low magnification images of the entire

the lowest expressing vector, AAV8 (double Y-F), we saw that use of AAV8 (double $\mathrm{Y}-\mathrm{F}+\mathrm{T}-\mathrm{V}$ ) resulted in the highest GFP intensity levels compared to unmodified AAV8 and AAV8 (double Y-F), (2612 a.u. vs. 1420 a.u. $p<0.01$ and 492 a.u. $p<0.0001$, respectively) (Fig. 2). Interestingly, we also noted that unmodified AAV8 was superior to AAV8 (double Y-F) $(p<0.05)$. These observations reflect a substantial difference in uptake kinetics and hints at some differences in trafficking kinetics. Taken together, we show that AAV8 (double $\mathrm{Y}-\mathrm{F}+\mathrm{T}-\mathrm{V}$ ) $>$ AAV8 $>$ AAV8 (double $\mathrm{Y}-\mathrm{F}$ ), therefore, all subsequent experiments focused on the use of AAV8 (double $\mathrm{Y}-\mathrm{F}+\mathrm{T}-\mathrm{V})$.

\section{Global brain biodistribution is achieved by modulating rAAV delivery route}

As IC6-based vector administration represents a relatively invasive procedure, we next sought to establish whether mid-sagittal section are shown below regional high magnification images. Images obtained using ScanScope FL. Cortex, thalamus, and cerebellum $(\times 20)$, scale bar $=100 \mu \mathrm{m}$; hippocampus $(\times 8)$, scale bar $=$ $300 \mu \mathrm{m}$.

comparable global brain biodistribution could be similarly achieved using alternate methods, namely ICV, thalamic (TH), and VTA, compared to IC6 with AAV8 (double Y-F $+\mathrm{T}-\mathrm{V}$ ) delivery (Fig. 3). Each method was previously demonstrated to be a well-tolerated, effective method to achieve widespread brain biodistribution. Both ICV and TH methods represent bilateral vector administration in only two injection sites, whereas the VTA method utilizes a single injection into the parenchyma.

Three months after rAAV injection, four spatially distinct and relatively equidistant areas were selected for qualitative and quantitative assessment of GFP expression. Using the Allen Brain Atlas, we estimated the relative anatomical locations, in millimeters, medial to lateral, to be -4.2 (section 1), -3.72 (section 2), -2.72 (section 3), and -1.72 (section 4), respectively. Overall percentage area with GFP expression for all four sections was initially compared using MPS IIIB mice. Compared to the IC6 method, no other method resulted in global 


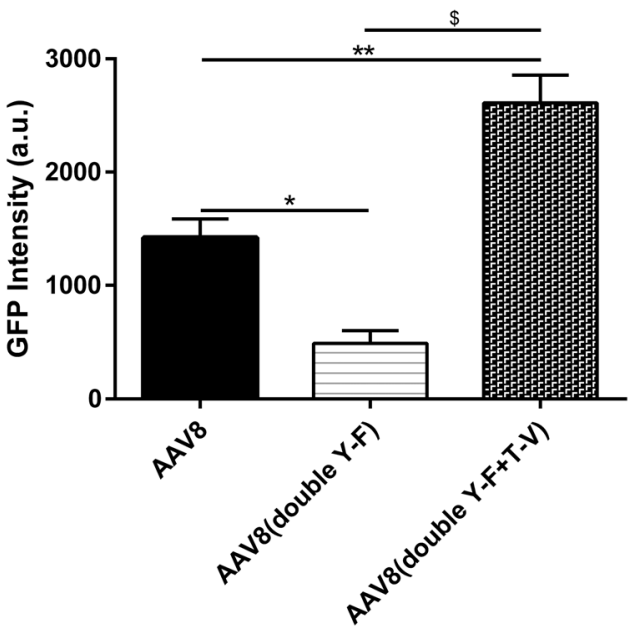

Fig. 2 Use of AAV8 (double $\mathrm{Y}-\mathrm{F}+\mathrm{T}-\mathrm{V}$ ) results in superior GFP intensity levels. Brains of 3-month-old MPS IIIB animals injected with AAV8, AAV8 (double Y-F), or AAV8 (double Y-F + T-V) via the IC6 method were collected and processed. Cortical, hippocampal, thalamic, and cerebellar limits of sagittal tissue sections were delineated and differences in mean GFP intensity levels across the regions for each animal and vector injected were assessed using the Image Studio Lite software and quantitated using one-way ANOVA. Data were normalized to AAV8 (double Y-F) GFP intensity. ${ }^{*} p<0.05$; $* * p<0.01 ;{ }^{\$} p<0.0001 ; n=4$.

tissue penetration and biodistribution. Of the three other methods, ICV-delivered rAAV showed relatively confined spread to the cortical area above the hippocampus, hippocampus, and moderate spread to the thalamus and cerebellum, although lesser penetration into other tissue sections was observed. The TH delivered similar overall GFP percentage area to ICV. VTA method was far lower by comparison.

In the IC6 treated group, AAV8 (double $\mathrm{Y}-\mathrm{F}+\mathrm{T}-\mathrm{V}$ ) transduction is observed throughout the cortex, hippocampus, caudate putamen, inferior, and superior colliculus and to a lesser degree, the thalamus and cerebellum. In the cortex, robust expression was observed throughout all layers associated with somatomotor and somatosensory cues, primarily layer V. Robust expression was also observed in all layers of the frontal cortex and in orbital areas of the prefrontal cortex. Both sensory- and motor-related areas of the superior colliculus exhibited high GFP expression. In the hippocampus, widespread GFP expression was observed in all layers. In the thalamus, areas proximal to the hippocampus were transduced (Fig. 3). Furthermore, resulting vector transduction of the cerebellum was modest and resulted in GFP expression in what appeared based on morphologic assessment to be purkinje neurons, but not interneurons.

Quantitatively, we see diminishing but substantial GFP expression in all tissue sections, overwhelmingly exhibited via the IC6 method, from $\sim 21 \%$ total GFP positive area in tissue section 1 , down to $\sim 6 \%$ in section 4 . It is important to note that overall, no other method of delivery achieves comparable levels of GFP biodistribution (IC6 vs. ICV and TH, $p<0.05$ for both, and IC6 vs. VTA, $p<0.01$ for all, Fig. 4). Taken together, we conclude that IC6 rAAV administration is still the method of choice to foster global brain biodistribution.

\section{Systemic transduction from CNS delivery of rAAV is observed}

As transport across the blood-brain barrier of some rAAV vectors has previously been reported, we sought to determine whether this phenomenon would also be apparent in the MPS IIIB model. We therefore assessed various somatic organs including the heart, liver, muscle, kidney, and spleen of AAV8 (double $\mathrm{Y}-\mathrm{F}+\mathrm{T}-\mathrm{V}$ ) treated MPS IIIB animals. We assessed for this phenomenon in IC6 and ICV treated animals as parenchymal transport may be less likely to occur than transport out of the ventricular spaces. Interestingly, we observed rAAV transduction into the heart and liver of treated animals with both methods, but did not observe muscle transduction (Fig. 5). Compared to the IC6 method, ICV administration of AAV8 (double Y-F + $\mathrm{T}-\mathrm{V}$ ) resulted in a higher perceived degree of organ transduction.

\section{Discussion}

In the present study, we investigated the impact of administration of AAV8 and capsid-mutant AAV8 vectors, AAV8 (double Y-F) and AAV8 (double $\mathrm{Y}-\mathrm{F}+\mathrm{T}-\mathrm{V}$ ), on the efficiency of brain transduction. To maximize therapeutic potential, we also investigated four brain administration methods: IC6, ICV, TH, and VTA for their effect on modulating global biodistribution. This is the first study that reveals the efficiency of capsid mutated AAV8 vectors in the CNS.

The efficiency of rAAV transduction is dependent on multiple steps involving virus-host cell interactions, which include binding to cellular receptors, overcoming intracellular barriers that limit nuclear accumulation of the virus and the conversion of single-stranded viral genomes to double-stranded forms [15]. As previously noted, the capsid is an essential element that influences both the extracellular events related to the recognition of specific receptors and intracellular processes affecting the trafficking and uncoating. Thus, the capsid plays an essential role in the cellular tropism, transduction kinetics, and intensity of efficiency of transgene expression $[6,16]$. Modulating these properties can improve both the effectiveness and safety of gene therapy. 


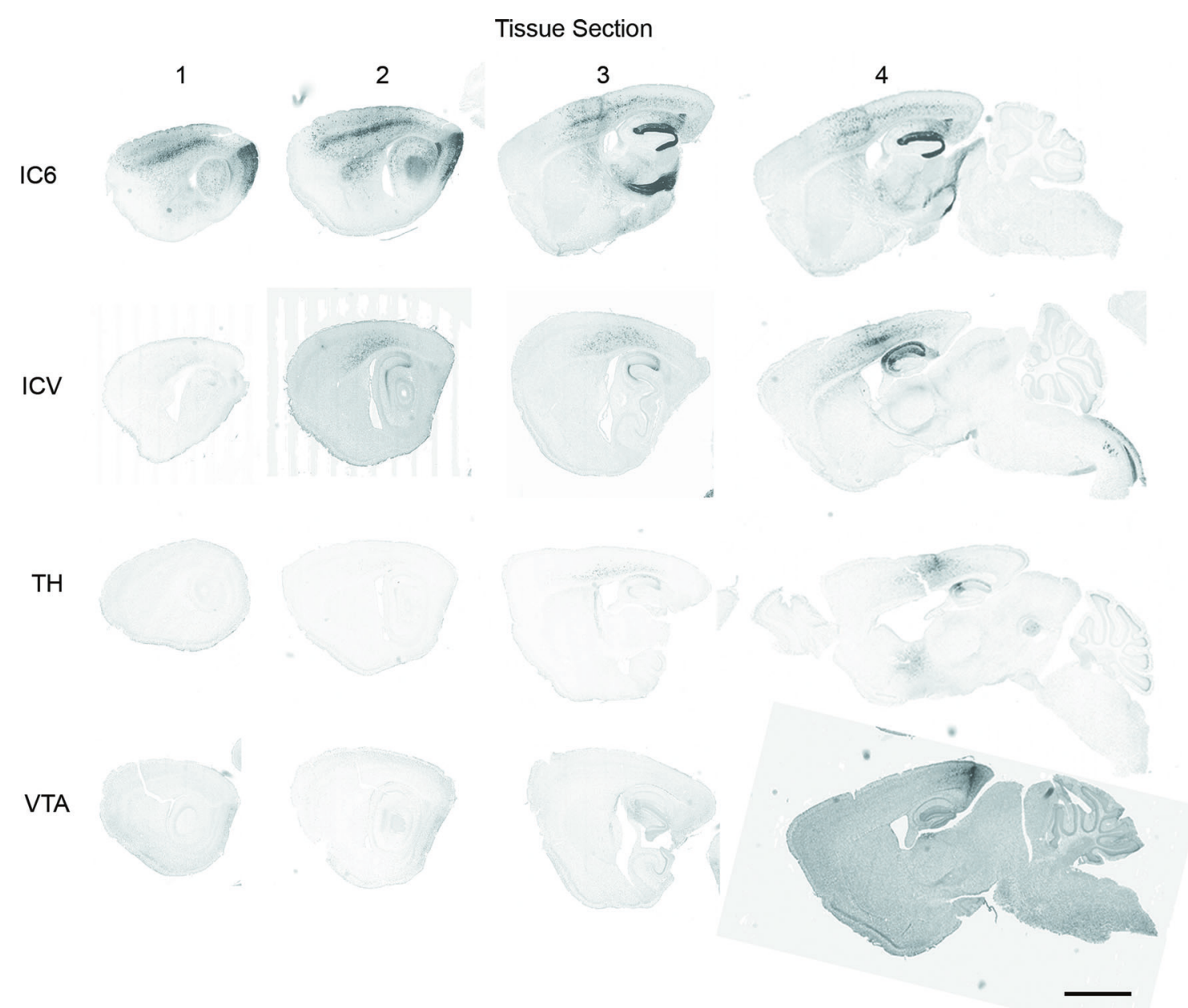

Fig. 3 The route of AAV administration impacts tissue penetration and biodistribution. AAV8 (double $\mathrm{Y}-\mathrm{F}+\mathrm{T}-\mathrm{V}$ ) was injected into neonatal MPS IIIB animals via the IC6, ICV, TH, and VTA methods. Brains were extracted at 3 months, sectioned into four structurally unique, relatively equidistant sections, in millimeters from

Tyrosine, serine, or threonine phosphorylation serves as a signal for ubiquitination of intact AAV particles, leading to subsequent targeting for the proteasome-mediated vector degradation before reaching the nucleus. In this context, mutation of capsid tyrosine and threonine residues is predicted to allow the vectors to escape the proteasome degradation pathway and thus promote more vector genome delivery to the nucleus and more effective transgene expression.

The increase in transduction efficiency gained from using capsid mutated vectors has been demonstrated in several disease models $[3,11,12,17,18]$. We have previously shown that IC6 administration of AAV8 leads to global biodistribution within the brain of neonatal MPS IIIB mice [13]. To investigate the potential benefit of using capsid modified vectors, we first selected available vectors based on AAV8, namely AAV8 (double Y-F) and AAV8 (double midline -4.2 (section 1), -3.72 (section 2), -2.72 (section 3), and -1.72 (section 4), respectively, and assessed for differences in tissue penetration and GFP biodistribution as a consequence of route of administration. Images obtained using ScanScope FL. Scale bar $=2 \mathrm{~mm}$.

$\mathrm{Y}-\mathrm{F}+\mathrm{T}-\mathrm{V}$ ), for comparison. Our results showed a significant and robust difference in transduction efficiency among these different vectors (Figs. 1 and 2). Although, AAV8 (double $\mathrm{Y}-\mathrm{F}+\mathrm{T}-\mathrm{V}$ ) emerged superior to AAV8 and AAV8 (double Y-F), we were surprised to find that AAV8 (double Y-F) performed worse than AAV8. Since both AAV8 (double Y-F) and AAV8 (double Y-F + T-V) are mutated at the same residues, it can be concluded that the $\mathrm{T}-\mathrm{V}$ substitution in AAV8 (double $\mathrm{Y}-\mathrm{F}+\mathrm{T}-\mathrm{V}$ ) plays an important role in modulating intracellular trafficking in neural cells. In comparing the effectiveness of the method of administration in fostering global brain biodistribution, we found that the IC6 method was far superior to the ICV, TH, and VTA methods (Figs. 3 and 4). In subjective review of the images for the various injection methods, MPS IIIB mice appeared to have higher transduction than WT with the both the AAV8 (double $\mathrm{Y}-\mathrm{F}+\mathrm{T}-\mathrm{V}$ ) and with native 
AAV8, in agreement with our prior publications also showing higher transduction with AAV8 in MPS IIIB than WT [14, 19].

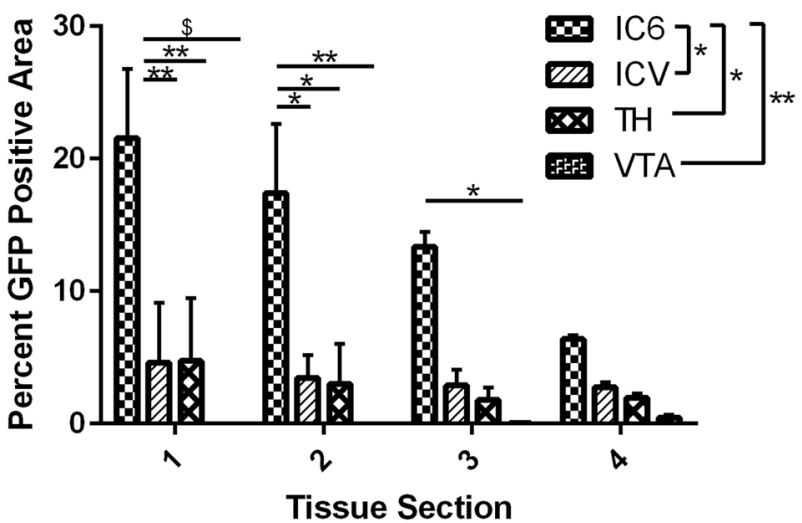

Fig. 4 The IC6 method of administration results in the most widespread GFP biodistribution within the brain. Using 3-monthold MPS IIIB animals, differences in GFP biodistribution as a consequence of method of administration were quantitatively assessed in each of the four tissue sections; as well as, cumulatively as indicated in the figure legend. Data were analyzed by two-way ANOVA. Data represented as mean \pm SEM. ${ }^{*} p<0.05 ; * * p<0.01 ;{ }^{\#} p<0.001 ;{ }^{\$} p<$ $0.0001 ; n=5$ for IC6 and $n=3$ each for ICV, TH, and VTA.
We cannot rule out the possibility that some of this effect may be related to differing total volume of injection. The maximum injection volume for our Hamilton syringe is $4 \mu \mathrm{l}$ and we were uncomfortable injecting more than this in any one site due to concerns for displacement of tissue and inducing further artifact by repeated needle placement at a site in order to reload the syringe and increase volume. We therefore adjusted concentrations of vector to fit the same total vector copy number/injection method in the volume we could comfortably inject with one syringe load for each site per method.

Given the unique distribution profiles associated with each method, we postulate that a disease-specific method of administration should be considered. For example, we saw that ICV method resulted in very high transduction of the hippocampus, and parts of the cortex and thalamus (Fig. 3). This method of administration may be more effective in the treatment of Alzheimer's, where severe pathology first affects parts of the cortex and the hippocampus. Similarly, we saw that a single VTA injection resulted in localized deposition in the mid-brain. This application may be better suited to the treatment of Parkinson's disease, which affects cells in a localized region, the substantia nigra. Furthermore, consistent with previous findings, we also demonstrate brain
Fig. 5 CNS administration of AAV8 (double Y-F + T-V) results in somatic transduction of organs. Three months after IC6 and ICV routes of AAV administration, somatic organs were harvested and assessed for presence of GFP. Both injection methods result in preferential transduction of heart and liver, indicating vector entry to systemic circulation. $\times 20$ images obtained with ScanScope FL. Scale bar $=300 \mu \mathrm{m}$.

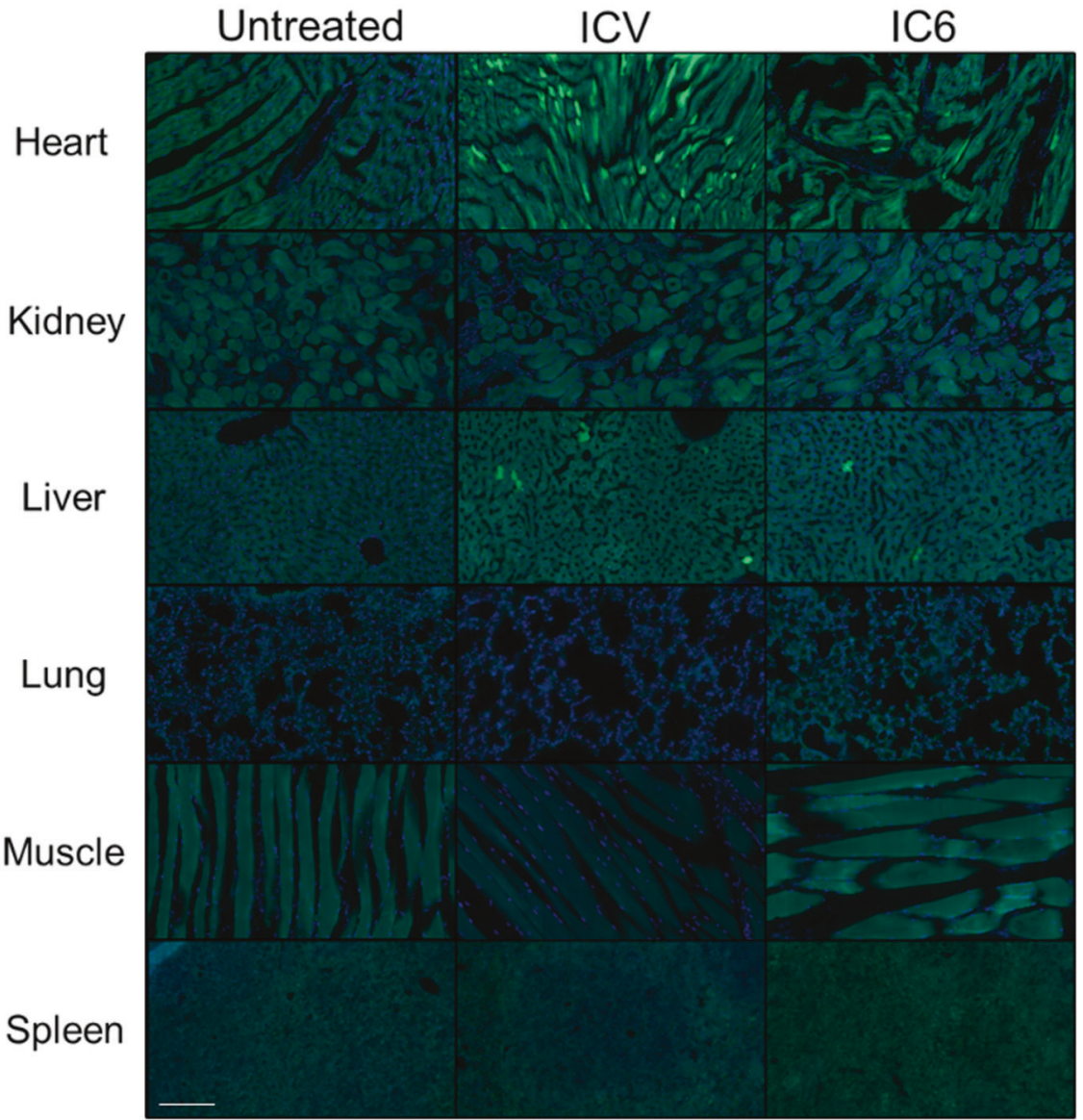


to systemic spread of AAV8 (double Y-F + T-V), which was more pronounced when administered via ICV delivery (Fig. 5).

In conclusion, the present study is the first to analyze the transduction capacity of AAV8 capsid tyrosine/threonine modified vectors and their impact on brain transduction in the MPS IIIB model. As successful treatment of MPS IIIB will require global vector biodistribution and tissue penetration, we also sought to identify an optimal method of delivery which would maximize therapeutic potential. Taken together, our data revealed that IC6 administration of an AAV8 (double $\mathrm{Y}-\mathrm{F}+\mathrm{T}-\mathrm{V}$ ) vector results in enhanced biodistribution of transgene expression in the CNS. It is expected that when combined with a codon optimized $\alpha-\mathrm{N}$ acetylglucosaminidase (NAGLU) this will result in increased therapeutic benefit, although the modest transport of vector into systemic circulation may warrant use of immunomodulatory agents.

\section{Materials and methods}

\section{Mice}

The congenic C57BL/6 NAGLU-deficient mouse strain was a kind gift from Elizabeth Neufeld (UCLA) by way of Mark Sands (Washington University, St. Louis, MO), and was maintained and expanded by strict sibling mating [20]. Wild type $(+/+)$, heterozygous $( \pm)$ (subsequently referred to as "Control") and mutant (-/-). Genotyping was done on tissue of newborn mice (P2-3) by enzyme assay [21] or NAGLU exon 6 and neomycin insertion cassette PCR. All animal studies were performed in accordance with guidelines of the University of Florida Institutional Animal Care and Use Committee.

\section{AAV constructs}

Recombinant AAV2 plasmids pseudotyped with capsid proteins from AAV8 were produced, purified, and titered at the University of Florida Powell Gene Therapy Center Vector Core Laboratory (Gainesville, FL) as previously described [22]. Vector titer was determined by dot blot assays, diluted, aliquoted, and stored at $-80^{\circ} \mathrm{C}$ until use.

Site-directed mutagenesis of surface-exposed tyrosine residues on AAV2 capsids has been described recently [6]. Similar strategies were used to generate AAV serotype 8 vectors containing tyrosine to phenylalanine mutations [23]. Vector preparations were produced using the plasmid cotransfection method.

Each of the three single-stranded rAAV vectors, AAV8, AAV8 (double Y-F), and AAV8 (double Y-F + T-V), express humanized green fluorescent protein driven by the hybrid cytomegalovirus enhancer/chicken beta-actin promoter and were kind gifts from Shannon Boye at the University of Florida.

\section{Treatments}

All treatments were performed in genotyped pups at 3-4 days of age and were well tolerated. For each comparison, 3-5 control and 3-5 MPS IIIB neonatal mice were injected by one of four methods; intracranial six site (IC6, $n=5)$, ICV $(n=3)$, thalamic (TH, $n=3)$, or VTA $(n=3)$. Neonates were cryoanesthetized prior to and during treatment and were then placed on a warming pad after treatment, before being returned to their mothers. All treatments were well tolerated. Intracranial rAAV-GFP was administered using the following coordinates determined by ruler: IC6-bilateral frontal (from bregma: $2 \mathrm{~mm}$ lateral, $1 \mathrm{~mm}$ posterior, and $1.5 \mathrm{~mm}$ deep), bilateral temporal (from bregma: $3 \mathrm{~mm}$ lateral, $3 \mathrm{~mm}$ posterior, and $2.5 \mathrm{~mm}$ deep), and bilateral cerebellar (from lambda: $1 \mathrm{~mm}$ lateral and posterior, $1.5 \mathrm{~mm}$ deep); $\mathrm{TH}$ (bilateral injections from bregma: $4 \mathrm{~mm}$ lateral, $1 \mathrm{~mm}$ posterior, and $3 \mathrm{~mm}$ deep); ICV - needle was placed perpendicular to the skull surface bilaterally $(0.25 \mathrm{~mm}$ lateral to the sagittal suture, $0.50-0.75$ $\mathrm{mm}$ rostral to the neonatal coronary suture, and $2 \mathrm{~mm}$ deep) [17]; and VTA (unilateral injection from bregma: $4.2 \mathrm{~mm}$ lateral and $4 \mathrm{~mm}$ deep; modified from Wolfe et al. [18]). All injections were conducted by hand through the skull using a 32-gauge Hamilton syringe (Narishige Int., East Meadow, NY). All mice received a total of $1.4 \times 10^{10}$ vector genomes in $4-12 \mu \mathrm{l}$ volumes over $1-3 \mathrm{~min}$.

\section{Histological procedures}

Animals were sacrificed 3 months after vector infusion. Mice were euthanized with $100 \mu \mathrm{l}$ of Ketamine $(120 \mathrm{mg} /$ $\mathrm{kg}) /$ Xylazine $(16 \mathrm{mg} / \mathrm{kg})$ cocktail followed by thoracotomy. Transcardial perfusion with $1 x$ PBS followed by fresh icecold $4 \%$ paraformaldehyde in 1xPBS solution followed. Brains were harvested, post fixed for $3 \mathrm{~h}$ in $4 \%$ paraformaldehyde at $4{ }^{\circ} \mathrm{C}$, followed by overnight incubation in $20 \%$ sucrose in $1 \times$ PBS at $4{ }^{\circ} \mathrm{C}$. One brain hemisphere was then embedded in OCT (Triangle Biomedical Sciences, Durham, NC) and rapidly frozen in a 2-methyl-butane/dry ice bath. Sagittal sections were cut to a thickness of $20 \mu \mathrm{m}$ and stored in a cryoprotective solution at $-80^{\circ} \mathrm{C}$ until use.

\section{Quantitation of GFP}

Quantitation of GFP positive area was conducted using the Scanscope FL instrument (Aperio Technologies, Vista, CA). Analysis was conducted using accompanying ImageScope software and the Positive Pixel Count FL v1 
algorithm. Use of the tuning feature allowed for maximal capture of GFP based on pixel intensity. The minimum intensity was set between 0.2 and 0.22 , and maximum intensity was set to 1 . Regions of interest were demarcated using the Allen Reference Atlas as a neuroanatomical reference. GFP positive area was determined using the average from three independent tests performed by three readers in a blinded manner.

To reliably assess differences in GFP intensity as a consequence of AAV capsid modification, the Odyssey Infrared Imaging system (Li-Cor, Lincoln, NE) was utilized. Briefly, mid-sagittal brain sections of AAV8, AAV8 (double $\mathrm{Y}-\mathrm{F}$ ), or AAV8 (double $\mathrm{Y}-\mathrm{F}+\mathrm{T}-\mathrm{V}$ ) vector treated animals were incubated overnight with GFP antibody (1:2000, Abcam, Cambridge, MA; Cat. \#: ab290) in $1 \times$ PBS $/ 0.01 \%$ TBS-T/10\% NDS/1\% BSA buffer. To visualize GFP, donkey anti-rabbit 680 DR was used (1:5000, Li-Cor Biosciences, Lincoln, NE, Cat. \# 926-68073). Sections are then mounted to slides and allowed to dry overnight followed by clearing with Xylene. Slide mounted sections were again air-dried overnight and scanned using the Odyssey system the following day. Analysis was conducted using the Image Studio Lite version 4.0 software (Li-Cor Biosciences, Lincoln, NE).

\section{Statistical analysis}

Sample sizes for groups were based on previously observed effect size in intensity of 4 and GFP percent area of 3 between AAV8 in control and MPS IIIB. In order to identify an effect size of 3.14 with alpha error of 0.05 and power of 0.8, a group size of three is required. GraphPad Prism 6 was used for statistical analysis. Two-tailed Student's $t$ test was used for unpaired data. Brown-Forsythe test was performed to confirm equal variance and Tukey's test was used to correct for multiple comparisons for ANOVA analysis. For comparisons with unequal variance, a log transform was used to normalize the data and reduce heterogeneity. Bar graphs are shown as mean \pm SEM. Probability $p<0.05$ was considered statistically significant. Animals were allocated to injection method by sequential selection of alternating method for each virus of animals randomly selected from each genotype. Allocation of areas of analysis for histology was carried out in a blinded fashion but the sample preparation and analysis itself was not blinded. From the intention-to-treat animals, those with evidence of vector extrusion during injection were excluded from analysis.

Acknowledgements This work was supported by the Gatorade Trust through funds distributed by the University of Florida, Department of Medicine and by K085141-01 and NIH/NINDS R01NS102624
(CDH), R01EY024280 (SEB) and R01HL-097088, R01GM-119186, and R21 EB-015684 (AS). We would like to express appreciation to Andrew Kolarich for assistance with performance of neonatal intracranial injections.

\section{Compliance with ethical standards}

Conflict of interest CDH, RJM, and AS declare stock ownership and co-founding of Lacerta Therapeutics, which develops gene therapy for CNS diseases. AS also holds several issued US Patents on AAV vectors that have been licensed to various gene therapy companies, and is a co-founder of Nirvana Therapeutics. SEB and SLB declare stock ownership and co-founding of Atsena Therapeutics, which develops gene therapies for ocular diseases.

Publisher's note Springer Nature remains neutral with regard to jurisdictional claims in published maps and institutional affiliations.

\section{References}

1. Di Natale P, Murino P, Pontarelli G, Salvatore D, Andria G. Sanfilippo B syndrome (MPS III B): altered residual alpha-Nacetylglucosaminidase activity in an unusual sibship. Clin Chim Acta. 1982;122:135-43.

2. Gilkes JA, Heldermon CD. Mucopolysaccharidosis III (Sanfilippo Syndrome)—disease presentation and experimental therapies. Pediatr Endocrinol Rev. 2014;12 Suppl 1:133-40.

3. Petrs-Silva H, Dinculescu A, Li Q, Min SH, Chiodo V, Pang JJ, et al. High-efficiency transduction of the mouse retina by tyrosinemutant AAV serotype vectors. Mol Ther. 2009;17:463-71.

4. Douar AM, Poulard K, Stockholm D, Danos O. Intracellular trafficking of adeno-associated virus vectors: routing to the late endosomal compartment and proteasome degradation. J Virol. 2001;75:1824-33.

5. Duan D, Yue Y, Yan Z, Yang J, Engelhardt JF. Endosomal processing limits gene transfer to polarized airway epithelia by adeno-associated virus. J Clin Investig. 2000;105:1573-87.

6. Zhong L, Li B, Jayandharan G, Mah CS, Govindasamy L, Agbandje-McKenna M, et al. Tyrosine-phosphorylation of AAV2 vectors and its consequences on viral intracellular trafficking and transgene expression. Virology. 2008;381:194-202.

7. Qing K, Hansen J, Weigel-Kelley KA, Tan M, Zhou S, Srivastava A. Adeno-associated virus type 2-mediated gene transfer: role of cellular FKBP52 protein in transgene expression. J Virol. 2001;75:8968-76.

8. Zhong L, Li B, Mah CS, Govindasamy L, Agbandje-McKenna M, Cooper M, et al. Next generation of adeno-associated virus 2 vectors: point mutations in tyrosines lead to high-efficiency transduction at lower doses. Proc Natl Acad Sci USA. 2008;105:7827-32.

9. Li M, Jayandharan GR, Li B, Ling C, Ma W, Srivastava A, et al. High-efficiency transduction of fibroblasts and mesenchymal stem cells by tyrosine-mutant AAV2 vectors for their potential use in cellular therapy. Hum Gene Ther. 2010;21:1527-43.

10. Markusic DM, Herzog RW, Aslanidi GV, Hoffman BE, Li B, Li $\mathrm{M}$, et al. High-efficiency transduction and correction of murine hemophilia B using AAV2 vectors devoid of multiple surfaceexposed tyrosines. Mol Ther. 2010;18:2048-56.

11. Martini SV, da Silva AL, Ferreira D, Gomes K, Ornellas FM, Lopes-Pacheco M, et al. Single tyrosine mutation in AAV8 vector capsid enhances gene lung delivery and does not alter lung morphofunction in mice. Cell Physiol Biochem. 2014;34:681-90. 
12. Qiao C, Zhang W, Yuan Z, Shin JH, Li J, Jayandharan GR, et al. Adeno-associated virus serotype 6 capsid tyrosine-tophenylalanine mutations improve gene transfer to skeletal muscle. Hum Gene Ther. 2010;21:1343-8.

13. Gilkes JA, Bloom MD, Heldermon CD. Mucopolysaccharidosis IIIB confers enhanced neonatal intracranial transduction by AAV8 but not by 5,9 or rh10. Gene Ther. 2015;23:263-71. https://doi. org/10.1038/gt.2015.111.

14. Gilkes JA, Bloom MD, Heldermon CD. Preferred transduction with AAV8 and AAV9 via thalamic administration in the MPS IIIB model: a comparison of four rAAV serotypes. Mol Genet Metab Rep. 2016;6:48-54.

15. Zolotukhin S, Potter M, Zolotukhin I, Sakai Y, Loiler S, Fraites TJ Jr, et al. Production and purification of serotype 1,2 , and 5 recombinant adeno-associated viral vectors. Methods. 2002;28:158-67.

16. Zolotukhin S, Byrne BJ, Mason E, Zolotukhin I, Potter M, Chesnut K, et al. Recombinant adeno-associated virus purification using novel methods improves infectious titer and yield. Gene Ther. 1999;6:973-85.

17. Gholizadeh S, Tharmalingam S, Macaldaz ME, Hampson DR. Transduction of the central nervous system after intracerebroventricular injection of adeno-associated viral vectors in neonatal and juvenile mice. Hum Gene Ther Methods. 2013;24:205-13.
18. Cearley CN, Wolfe JH. A single injection of an adeno-associated virus vector into nuclei with divergent connections results in widespread vector distribution in the brain and global correction of a neurogenetic disease. J Neurosci. 2007;27:9928-40.

19. Gilkes JA, Bloom MD, Heldermon CD. Mucopolysaccharidosis IIIB confers enhanced neonatal intracranial transduction by AAV8 but not by 5, 9 or rh10. Gene Ther. 2016;23:263-71.

20. Li HH, Yu WH, Rozengurt N, Zhao HZ, Lyons KM, Anagnostaras $\mathrm{S}$, et al. Mouse model of Sanfilippo syndrome type B produced by targeted disruption of the gene encoding alphaN-acetylglucosaminidase. Proc Natl Acad Sci USA. 1999;96: 14505-10.

21. Marsh J, Fensom AH. 4-Methylumbelliferyl alpha-Nacetylglucosaminidase activity for diagnosis of Sanfilippo B disease. Clin Genet. 1985;27:258-62.

22. Sen D, Gadkari RA, Sudha G, Gabriel N, Kumar YS, Selot R, et al. Targeted modifications in adeno-associated virus serotype 8 capsid improves its hepatic gene transfer efficiency in vivo. Hum Gene Ther Methods. 2013;24:104-16.

23. Li C, Diprimio N, Bowles DE, Hirsch ML, Monahan PE, Asokan A, et al. Single amino acid modification of adeno-associated virus capsid changes transduction and humoral immune profiles. J Virol. 2012;86:7752-9. 